\title{
Alterations in VEGF expression induced by antidepressant drugs in female rats under chronic social stress
}

\author{
MARTA MARIA NOWACKA-CHMIELEWSKA ${ }^{1,2}$, MONIKA PAUL-SAMOJEDNY ${ }^{3}$, \\ ANNA MARIA BIELECKA-WAJDMAN ${ }^{4}$, JAROSŁAW JERZY BARSKI ${ }^{2,5}$ and EWA OBUCHOWICZ ${ }^{4}$
}

${ }^{1}$ Laboratory of Molecular Biology, Faculty of Physiotherapy, The Jerzy Kukuczka Academy of Physical Education, 40-065 Katowice; ${ }^{2}$ Center For Experimental Medicine, School of Medicine in Katowice, Medical University of Silesia, 40-752 Katowice; ${ }^{3}$ Department of Medical Genetics, School of Pharmacy with The Division of Laboratory Medicine, Medical University of Silesia, 41-200 Sosnowiec; Departments of ${ }^{4}$ Pharmacology and ${ }^{5}$ Physiology, School of Medicine in Katowice, Medical University of Silesia, 40-752 Katowice, Poland

Received February 23, 2015; Accepted July 17, 2016

DOI: $10.3892 /$ etm.2017.4022

\begin{abstract}
Vascular endothelial growth factor (VEGF) is thought to serve a role in neurogenesis and the stress response. Although a definite link between the action of antidepressants and VEGF has not been identified, it is assumed that VEGF, as a neurotrophic factor, serves an important role in the effects of antidepressant treatment. To examine this, the present study subjected adult female rats to four weeks of social instability stress and measured the effect of antidepressant treatment on the expression of VEGF. Firstly, endocrine markers of stress and body weight were measured in parallel with behavioral tests prior to and following subjection to stress. Then, the effect of 28-day daily treatment with desipramine (DMI; $10 \mathrm{mg} / \mathrm{kg}$ ), fluoxetine $(5 \mathrm{mg} / \mathrm{kg})$ or tianeptine $(10 \mathrm{mg} / \mathrm{kg})$ on the number of copies of VEGF mRNA in the amygdala, hippocampus and hypothalamus, and on serum VEGF protein levels, of rats subjected to chronic stress was determined. In addition, the weight of the adrenal glands was measured following subjection to stress. Exposure to chronic stress was found to increase the rats' sucrose preference, and diminish their tendency for general exploration and time spent in the open. The relative adrenal weights of the stressed rats were significantly increased compared with the control. Plasma concentrations of corticosterone and adrenocorticotropic hormone were not significantly augmented. In addition, the present study identified that stress elevated VEGF mRNA expression in all studied neural structures. Furthermore, the results identified that the stress-induced increase in VEGF mRNA expression in
\end{abstract}

Correspondence to: Dr Marta Maria Nowacka-Chmielewska, Laboratory of Molecular Biology, Faculty of Physiotherapy, The Jerzy Kukuczka Academy of Physical Education, 72a Mikolowska, 40-065 Katowice, Poland

E-mail: m.nowacka@awf.katowice.pl

Key words: antidepressants, blood, brain structures, chronic stress, female rats, vascular endothelial growth factor the amygdala and hypothalamus was attenuated by long-term administration of DMI. Conversely, a decrease in serum VEGF concentration was observed in stressed rats, which was not reversed by treatment with antidepressants. In conclusion, the current study suggests that under conditions of stress, VEGF serves a role in the mechanism of action of DMI, through modulating activity of the norepinephrine system.

\section{Introduction}

In recent years, vascular endothelial growth factor (VEGF) has been a primary subject of psychiatric research, with an emphasis on its potential role in major depressive disorder, a stress-related disease $(1,2)$. Chronic stress, which can be caused by social conflict or instability, serves a primary role in the provocation of depression, particularly in the presence of genetic vulnerability factors (3). It has been suggested that VEGF could be dysregulated under stress and in depressive states (4).

VEGF, initially identified as an angiogenic mitogen, is thought to serve as a neurotrophic factor in hippocampal neurogenesis (5) and influence synaptic transmission (6). VEGF regulates neurite outgrowth and maturation during development (7), and in the adult brain can influence a number of complex processes, including learning and memory (8).

Since VEGF emerged as a putative factor in the pathogenesis of stress-related disorders and the efficacy of antidepressive therapy (9-11), experimental studies have been conducted to delineate its precise role $(9,10)$. A definitive link between the action of antidepressants and VEGF has yet to be elucidated, however, it is assumed that as a neurotrophic factor VEGF serves an important role in the effects of antidepressants. The neurogenic/neurotrophic hypothesis of depression implicates a stress-induced decline in neurotrophins, such as brain-derived neurotrophic factor (BDNF) and VEGF, and atrophy of the limbic structures in the pathogenesis of mood disorders $(12,13)$. A previous study in animal models of depressive-like behavior suggest that antidepressants increase VEGF levels, opposing the effect of stress (14). However, to the best of our knowledge, the influence of long-term antidepressant administration on 
hippocampal VEGF levels in rats exposed to chronic stress has been investigated in few studies and performed only in males (15-18).

Irrespective of environmental influences, such as chronic social stress, gender is a primary risk factor for major depression, with women having double the risk of developing affective disorders compared with men (19). Previous studies have shown that the physiological and behavioral responses of an organism to environmental factors are related to gender $(20,21)$. The present study, to the best of our knowledge, is the first to investigate the effect of long-term administration of antidepressants on the expression of VEGF mRNA in female rats exposed to chronic social stress. The effects of the tricyclic antidepressant desipramine, the selective serotonin reuptake inhibitor (SSRI) fluoxetine and the atypical antidepressant tianeptine were measured in structures implicated with emotional state and stress response regulation, the amygdala, hippocampus and hypothalamus. In addition, alterations in serum VEGF concentration elicited by chronic social stress and antidepressants were evaluated.

\section{Materials and methods}

Experimental animals. Adult female Sprague-Dawley rats $(\mathrm{n}=71$; age, 9 weeks) from the Centre For Experimental Medicine (Medical University of Silesia, Katowice, Poland) weighing between 200 and $220 \mathrm{~g}$ were used in the present study. Rats were kept under standard conditions (light/dark cycle, $12 \mathrm{~h}$; temperature, $20-24^{\circ} \mathrm{C}$; humidity, 45-65\%) with ad libitum access to food and water. All animal procedures were performed in accordance directive 2010/63/EU of the European Commission (22) and were approved by the Local Ethics Committee for the Care and Use of Laboratory Animals (Medical University of Silesia, Katowice, Poland).

Chronic social instability stress procedure. Rats were exposed to chronic social instability stress using a previous described procedure (23). Briefly, the procedure was based on alternating phases of isolation and crowding over 4 weeks. During isolation rats were housed individually in standard cages $(36 \times 20 \times 15 \mathrm{~cm})$, whilst during crowding rats were housed in groups of four in small cages $(30 \times 20 \times 15 \mathrm{~cm})$. To enhance unpredictability, different rats were housed together during each crowding phase. Control (non-stressed) rats were kept in groups of seven in standard cages $(52 \times 31 \times 19 \mathrm{~cm})$ in a separate room.

Treatments and experimental design. Stressed and control rats were injected intraperitoneally once daily between 7 and 9 a.m. for 28 days with an antidepressant drug (described below) or $2 \mathrm{ml} / \mathrm{kg} 0.9 \%$ saline (SAL). The following antidepressant drugs were used: $10 \mathrm{mg} / \mathrm{kg}$ desipramine hydrochloride (DMI; Sigma-Aldrich, St. Louis, MO, USA), $5 \mathrm{mg} / \mathrm{kg}$ fluoxetine hydrochloride (FLX; PLIVA, Ltd., Krakow, Poland) and $10 \mathrm{mg} / \mathrm{kg}$ tianeptine sodium salt (TIA; Servier Laboratories, Suresnes, France). Rats were weighed twice a week. Animals were randomly assigned to into the following five groups: Control $+\mathrm{SAL}$, unstressed rats treated with SAL ( $=7)$; stress + SAL, stressed rats treated with SAL $(n=8)$; stress + DMI, stressed rats treated with DMI $(n=8)$; stress + FLX, stressed rats treated with FLX $(n=8)$; and stress + TIA, stressed rats treated with TIA $(n=8)$.

The effect of chronic social stress on rat behavior, body weight, relative adrenal weight and endocrine markers of stress were studied on the following separate groups of rats: Control + SAL $(n=16)$; and stress + SAL $(n=16)$ using the sucrose preference test and open field test. An overview of the experimental design is described in Fig. 1A and B.

Collection of neural structures, adrenal glands, plasma and serum. Rats were sacrificed by decapitation $24 \mathrm{~h}$ following the last dose of the antidepressant or saline. Following sacrifice, the brain and the amygdala, hippocampus and hypothalamus were dissected according to the Paxinos and Watson atlas (24). Then, isolated brain structures were sonicated in ice-cold TRI Reagent (Sigma-Aldrich; $1 \mathrm{ml} / 100 \mathrm{mg}$ of tissue) and stored at $-80^{\circ} \mathrm{C}$ until required. Subsequent to the decapitation of rats and opening of the abdominal cavity, both adrenal glands were resected, weighed after careful removing of adherent fat, and then discarded. Furthermore, trunk blood (plasma and serum) samples were collected. To obtain plasma, blood was collected into pre-chilled tubes containing EDTA, whilst to acquire serum, blood was kept at room temperature for $30 \mathrm{~min}$ in tubes without anticoagulant. Following centrifugation $(10 \mathrm{~min}$, $2,500 \times g, 4^{\circ} \mathrm{C}$ ), the resulting supernatants, designated plasma and serum were aliquoted and stored at $-80^{\circ} \mathrm{C}$.

Sucrose preference test. The sucrose preference test, used for determination of anhedonic-like behavior, was conducted for $24 \mathrm{~h}$ on the day prior to and on the last day of the chronic stress procedure, according to a protocol described previously (23). Briefly, rats could choose between a bottle containing water or a bottle containing $1 \%$ sucrose solution. Rats had ad libitum access to food and water prior to, during and following the test. Control and stressed rats were tested individually. Sucrose preference (\%) was calculated as: Sucrose consumption $(\mathrm{ml}) /[$ (sucrose + water consumption $(\mathrm{ml})] \times 100$.

Open field test. The open field test was performed to measure locomotor activity and anxiety-related behavior. The test was conducted prior to and following the chronic stress protocol (the day following the sucrose preference test) using a transparent plastic chamber with a light grey floor $(55 \times 55 \times 40 \mathrm{~cm})$ in a softly lit experimental room. Locomotor activity of the rats, indicated by the speed and distance of their movements, was monitored by a computerized video-tracking system using Ethovison XT software (version 8.0; Noldus Information Technology B.V., Wageningen, The Netherlands). Rats were placed one at a time in the left corner of the 'open field' and recorded for $15 \mathrm{~min}$. Horizontal activity was measured in $\mathrm{cm}$ and vertical activity as the total rearing duration time. The percentage of time spent in the center of the field [(time spent in center) / (time spent in center + margin) $x$ 100] was used as an indication of anxiety. The floor surface of each chamber was thoroughly cleaned between the testing of each animal.

Corticosterone and adrenocorticotropic hormone (ACTH) assays. Plasma samples were collected as described previously. Adrenal glands were dissected and weighed as described above. The results are represented as percentage of body weight 
on the last experimental day. Plasma corticosterone concentrations were determined by ELISA using a Corticosterone ELISA kit (cat no. 501320; Cayman Chemical Company, Ann Arbor, MI, USA), according to the manufacturer's protocol. The results were determined by measuring the absorbance at $450 \mathrm{~nm}$ using a multiwell plate reader (Multiskan RC, LabSystems, Inc.; Thermo Fisher Scientific, Waltham, MA, USA). Plasma ACTH levels were determined with an automated chemiluminescent immunometric assay on an IMMULITE 2000 analyzer (Siemens Healthcare Diagnostics GmbH, Erlangen, Germany).

Total RNA extraction and reverse transcription-quantitative polymerase chain reaction ( $R T-q P C R)$. Total RNA was extracted from brain structures and $10 \mathrm{ng} / \mu \mathrm{l}$ was used for RT-qPCR according to previously described protocols $(25,26)$ to determine the levels of VEGF mRNA. The quality and integrity of the RNA extracted was assessed by $1 \%$ agarose gel electrophoresis and the optical density (260:280 $\mathrm{nm}$ ratio) was measured using a BioPhotometer (Eppendorf, Hamburg, Germany). TaqMan Gene Expression Assay primers and probes for amplification of VEGF mRNA were obtained from Thermo Fisher Scientific, Inc. RT-qPCR was performed using TaqMan One-Step RT-PCR Master Mix Reagents and the ABI Prism 7700 Sequence Detection system (both Thermo Fisher Scientific, Inc.). RT was performed at $48^{\circ} \mathrm{C}$ for $30 \mathrm{~min}$ and the following steps were performed for PCR: 40 cycles of $10 \mathrm{~min}$ at $95^{\circ} \mathrm{C} ; 1$ cycle at $95^{\circ} \mathrm{C}$ for $15 \mathrm{sec}$; and 1 cycle at $60^{\circ} \mathrm{C}$ for $1 \mathrm{~min}$. All experiments were performed in triplicate and rat GAPDH was used as an endogenous control. The copy numbers of VEGF mRNA were calculated using the Cq-based calibrated standard curve method for the $\beta$-actin standards (27) (TaqMan DNA Template Reagents, Scientific, Inc.) run during the same experiment.

Serum VEGF measurement. Quantification of VEGF in the sera was performed with an ELISA kit (cat no. RRV00; R\&D Systems, Inc., Minneapolis, MN, USA) according to the manufacturer's protocol. The results were determined by measuring the optical density at $450 \mathrm{~nm}$ using a multiwell plate reader (Multiskan RC; Thermo Fisher Scientific, Inc.).

Statistical analysis. GraphPad Prism software (version 6; GraphPad Software, Inc., La Jolla, CA, USA) was used for statistical and graph production. Differences in the adrenal weights and hormone measurements between groups were analyzed using unpaired t-tests. Analysis of behavioral data (the sucrose preference and open field tests) performed with a paired t-test or Wilcoxon signed-rank test. The statistical significance of differences between treatment groups was evaluated using a one-way analysis of the variance (ANOVA) followed by a Tukey's range test. $\mathrm{P}<0.05$ was considered to indicate a statistically significant difference. Results are presented as the mean \pm standard error of the mean.

\section{Results}

Behavioral tests. The average consumption of sucrose solution increased by $16 \%$ in chronically stressed rats treated with saline compared with unstressed rats treated with saline, and this difference was significant $(\mathrm{P}=0.001$; Fig. 2A).
In regards to locomotor activity, the stressed rats showed slightly reduced general locomotor activity. There was a $15.5 \%$ decrease in the distance moved $(\mathrm{P}=0.15)$ and velocity of movement $(\mathrm{P}=0.12)$ in the stressed rats compared with the controls (result not shown). It was also noted that the stressed animals showed reduced exploratory activity in the open field. Chronic stress significantly reduced vertical locomotor activity (rearing) of rats by $63 \%$ compared with the control $(\mathrm{P}=0.007$; Fig. 2B). In addition, rats subjected to chronic stress spent $34.6 \%$ less time in the center of the open field (plastic chamber) compared with control rats $(\mathrm{P}=0.09$; Fig. $2 \mathrm{C})$.

Weight gain, adrenal weight and hormone levels. Over the 4-week experimental period, the body weight of the stressed female rats increased by $\sim 24 \%$. At the end of the experiment, there was no significant difference in body weight gain in unstressed saline-treated rats $(279.4 \mathrm{~g})$ or stressed saline-treated rats $(294.0 \mathrm{~g})$ (results not shown). In addition, the relative (mg/g body weight) adrenal weight of the stressed rats was significantly increased compared with the control $(\mathrm{P}<0.0001$; Fig. 3A). In regards to hormone levels, the plasma corticosterone concentration in chronically stressed rats increased by 26\% ( $\mathrm{P}=0.30$; Fig. 3B) and ACTH concentration increased by $46 \%(\mathrm{P}=0.095$; Fig. 3C) compared with unstressed rats.

Influence of long-term antidepressant treatment on VEGF $m R N A$ expression in the brain structures of female rats subjected to chronic stress. In the hippocampus, chronic social instability stress increased the number of VEGF mRNA copies by $124 \%$ relative to the control (one-way ANOVA, $\mathrm{P}=0.0054$; Tukey's range test, $\mathrm{P}<0.05)$. In stressed rats treated with $\mathrm{DMI}$, FLX or TIA, VEGF mRNA expression was increased by 53, 29 and $35 \%$, respectively, compared with the saline-treated stressed group, but these effects did not reach statistical significance. However, VEGF mRNA expression increased following treatment with all the studied antidepressants by 243\% (DMI: $\mathrm{P}<0.01$ ), 191\% (FLX: $\mathrm{P}<0.05$ ) or $204 \%$ (TIA: $\mathrm{P}<0.05)$ compared with the unstressed saline-treated control (Fig. 4A).

In the amygdala of stressed rats, there was a significant $85 \%$ increase in the VEGF expression compared with the control group (one-way ANOVA, $\mathrm{P}=0.015$; Tukey's range test: $\mathrm{P}<0.05$ ). In stressed rats treated with DMI or FLX, the VEGF expression decreased by 43 and $22 \%$, respectively, compared with the saline-treated stressed group, but these effects did not reach statistical significance. In DMI-treated rats, VEGF expression returned to the value found in the non-stressed control group. TIA had no effect on the VEGF expression in rats subjected to stress compared with stressed saline-treated rats (Fig. 4B).

In the hypothalamus, exposure to chronic stress elicited a significant increase of $64 \%$ in VEGF expression compared with the unstressed control rats (one-way ANOVA, $\mathrm{P}=0.0044$; Tukey's range test, $\mathrm{P}<0.05)$. In the DMI-treated stressed rats, VEGF expression was significantly reduced by $35 \%$ compared with saline-treated stressed rats $(\mathrm{P}<0.05)$. Similar to in the amygdala, FLX reduced VEGF expression by $24 \%$ compared with saline-treated stressed rats. VEGF expression in TIA-treated stressed rats did not differ from that in the saline-treated stressed rats, but was similarly significantly increased by $68 \%$ compared to the unstressed control $(\mathrm{P}<0.05$; Fig. $4 \mathrm{C})$. 
A

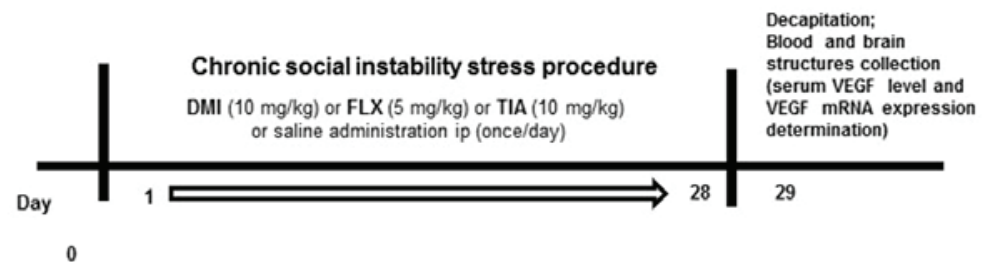

B

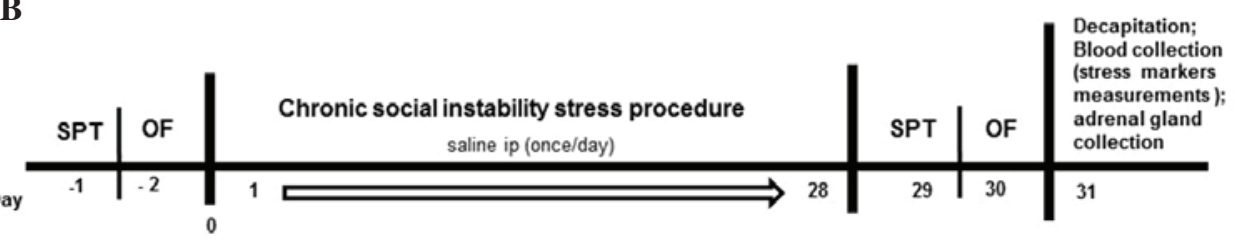

Figure 1. Experimental design. Antidepressant treatment was initiated concurrently with the chronic social instability stress procedure. DMI (10 mg/kg), FLX (5 mg/kg), TIA (10 mg/kg) or saline were administered ip once daily for 28 days. (A) Rats were sacrificed $24 \mathrm{~h}$ following the last dose of antidepressant or saline. A separate group of saline-treated rats were subjected to the behavioral tests (SPT and OF) prior to and following the chronic social instability stress procedure. (B) Rats were sacrificed $24 \mathrm{~h}$ following the OF. SPT, sucrose preference test; OF, open field test; DMI, desipramine; FLX, fluoxetine; TIA, tianeptine; ip, intraperitoneally.

A

Sucrose preference test

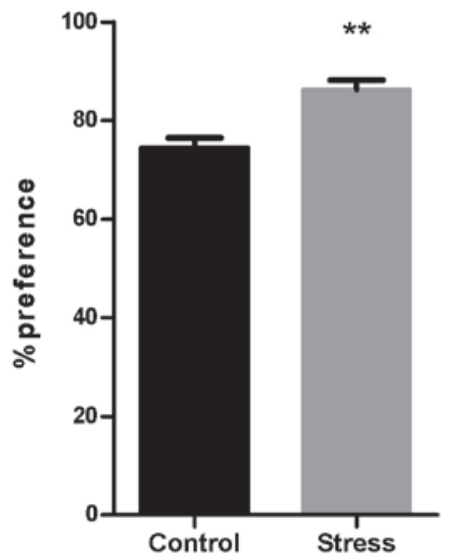

B

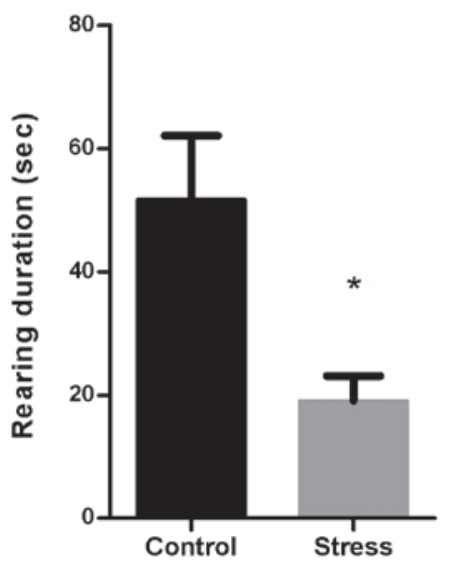

C

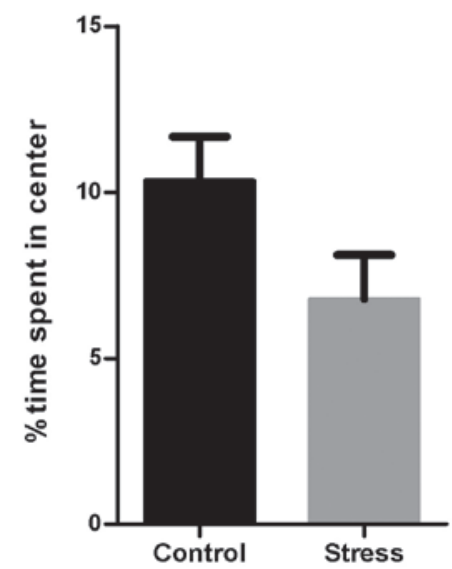

Figure 2. Effect of chronic social instability stress the on behavior of female rats. (A) Sucrose preference test. (B) Vertical movement (rearing duration) in the open field test. (C) Percentage of time spent in the center of the open field. Control group, $n=16$; stressed group, $n=16$. Both unstressed control and stressed groups were treated with saline. Data is presented as the mean \pm standard error of the mean. Differences between groups were statistically analyzed using a paired t-test. ${ }^{*} \mathrm{P}=0.007,{ }^{* *} \mathrm{P}=0.001$ vs. control.

A

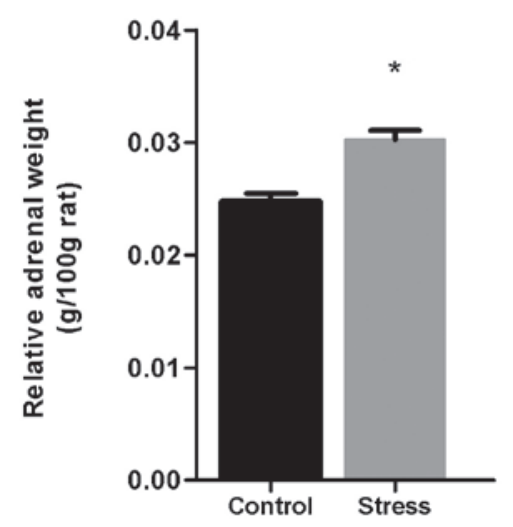

B

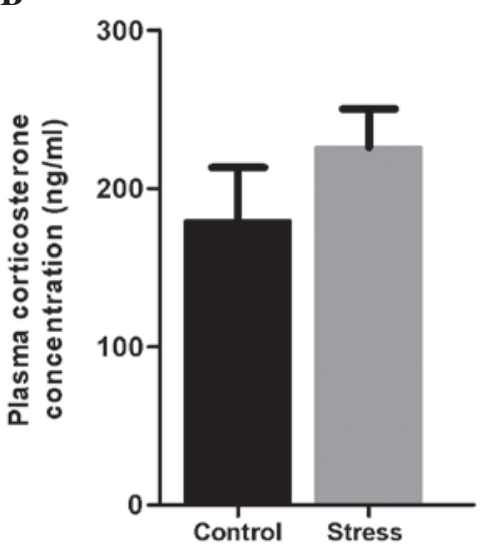

C

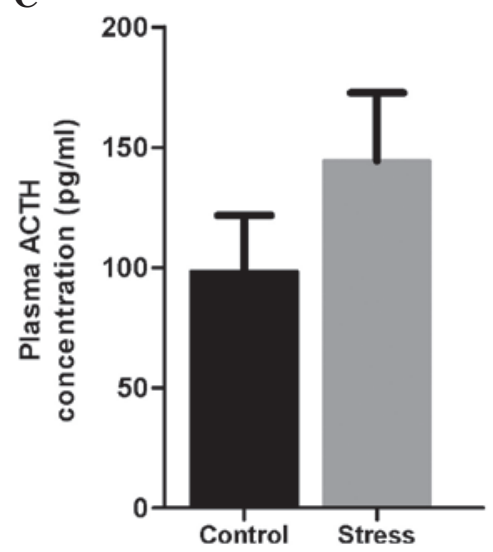

Figure 3. Effect of chronic social instability stress on adrenal gland weight and stress hormone levels in female rats. (A) The relative adrenal weight (mg/g body weight) of the control and stress groups treated with saline following the chronic social instability stress procedure. Plasma concentrations of (B) corticosterone and (C) ACTH following the chronic social instability stress procedure. Control group, $n=7$; stressed group, $n=8$. Data is presented as the mean \pm standard error of the mean. Differences between groups were statistically analyzed using unpaired t-tests. ${ }^{\prime \prime} \mathrm{P}<0.0001$ vs. control. ACTH, adrenocorticotropic hormone. 
Effect of long-term antidepressant treatment on serum VEGF protein concentration in female rats subjected to chronic stress. As shown in Fig. 5, exposure to chronic social stress significantly reduced serum VEGF levels by $25 \%$ compared to the control (one-way ANOVA, $\mathrm{P}<0.0001$; Tukey's range test, $\mathrm{P}=0.002)$. Serum VEGF concentration in DMI-treated rats increased by $20 \%$ compared with saline-treated stressed rats $(\mathrm{P}=0.097)$. There was no difference between the saline-treated and FLX-treated stressed groups. Although, in the FLX-treated stressed group protein VEGF levels significantly decreased by $30 \%$ compared with the unstressed saline-treated controls $(\mathrm{P}<0.01)$. Administration of TIA to stressed rats significantly diminished the level of VEGF by $40 \%$ compared with unstressed saline-treated control rats $(\mathrm{P}<0.001)$, and by $22 \%$ in comparison to the saline-treated stressed rats (no significant difference).

\section{Discussion}

The present study provides novel information regarding the effect of antidepressants on VEGF expression in stressed female rats. Firstly, chronic social instability stress was identified to increase the expression of VEGF mRNA in all the studied brain structures: the hippocampus, amygdala and hypothalamus. This supports the view that VEGF serves a role in brain function during chronic stress conditions and in the development of affective disorders. Secondly, the results of the current study indicate that long-term DMI or FLX, but not TIA, treatment attenuates the effect of stress on VEGF mRNA expression in the amygdala and hypothalamus. Surprisingly, in the hippocampus, all studied antidepressants increased VEGF mRNA expression. Finally, a decrease in sera VEGF protein concentration was identified in stressed rats, which antidepressant treatment did not reverse.

In order to evaluate the rat model of chronic social instability stress used in the present study, the impact on the hypothalamic-pituitary-adrenal axis and the behavior of rats was tested, using endocrine stress markers (adrenal weights, $\mathrm{ACTH}$ and corticosterone concentrations) and two behavioral tests (sucrose preference and open field tests). This evaluation was limited, as it was studied in the control + SAL and the stress + SAL groups, but not in the antidepressant treatment groups. The results determined that the adrenal glands of female rats exposed weighed more compared with control rats. Plasma concentrations of the stress hormones (corticosterone and $\mathrm{ACTH}$ ) were unaffected by the chronic social stress procedure used, which may suggest habituation to the procedure.

It is well established that chronic stress induces adaptive physiological and psychological processes, which may develop into a pathological response, particularly when the stress is sustained and coping attempts are unsuccessful (28). Unlike numerous previous reports demonstrating a reduction in sucrose intake by stressed rats $(29,30)$, the results of the present study indicate that the applied stress model induced the opposite effect. However, other studies on anhedonia following the application of mild stressors found similar results $(15,31)$.

The open field test was performed in the present study, in order to determine if the applied stress procedure induced behavioral changes in the rats. The results showed that
A
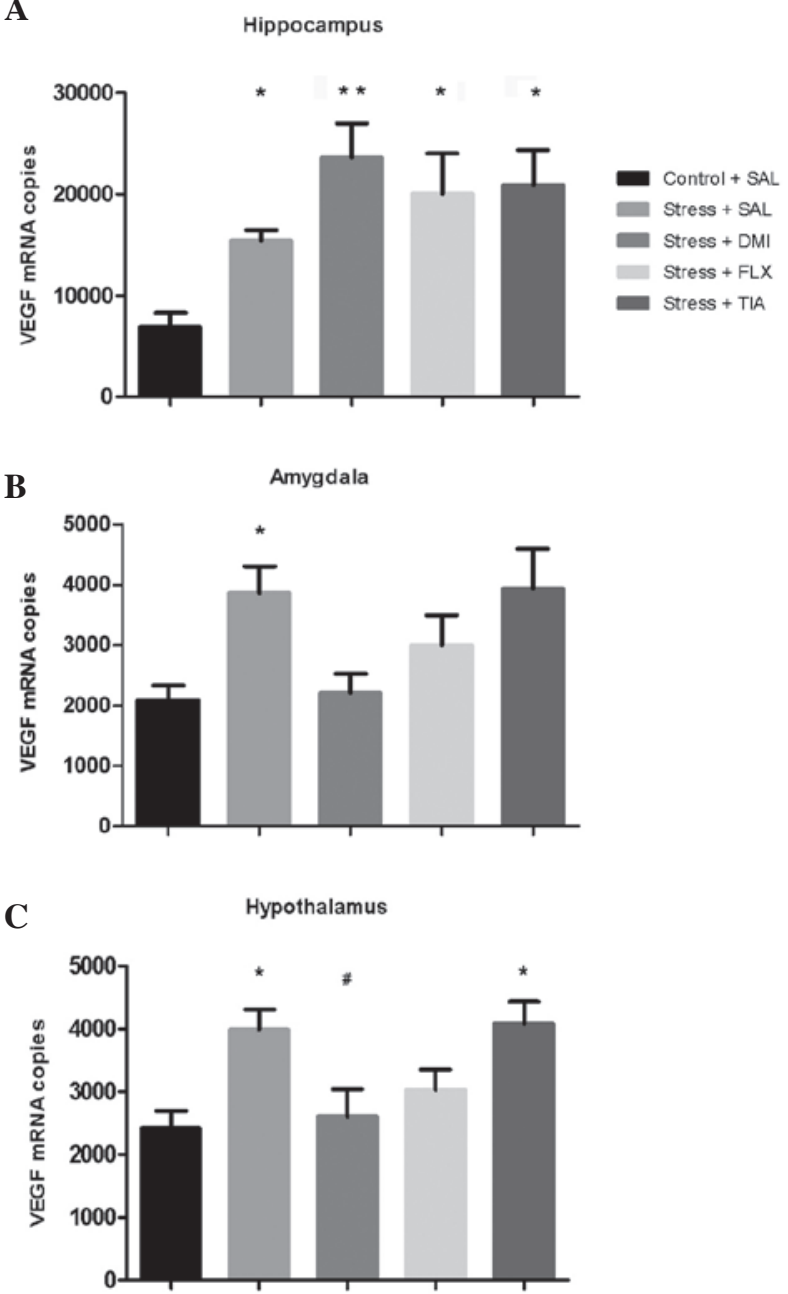

Figure 4. The effect long-term DMI, FLX or TIA treatment on the expression of VEGF mRNA copies in the (A) hippocampus, (B) amygdala and (C) hypothalamus of female rats subjected to chronic social instability stress. Data is presented as the mean \pm standard error of the mean. Differences between groups were statistically analyzed using one-way analysis of the variance followed by a Tukey's range test. ${ }^{*} \mathrm{P}<0.05,{ }^{* *} \mathrm{P}<0.01$ vs. control; ${ }^{\#} \mathrm{P}<0.05$, vs. stress $+\mathrm{SAL}$. VEGF, vascular endothelial growth factor; mRNA, messenger ribonucleic acid; SAL, saline; DMI, desipramine; FLX, fluoxetine; TIA, tianeptine.

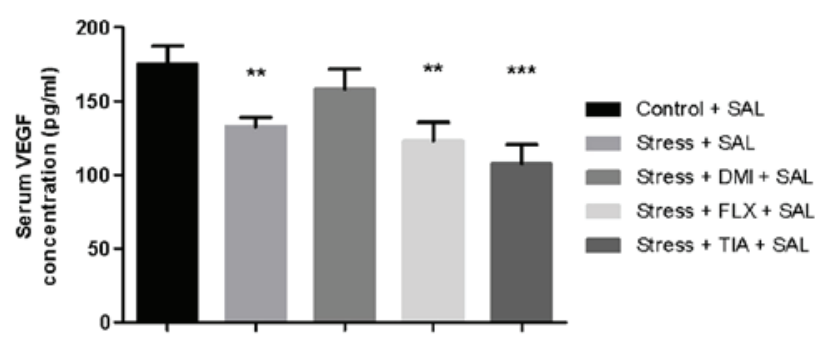

Figure 5. The effect long-term DMI, FLX or TIA treatment on the serum VEGF concentration of female rats subjected to chronic social instability stress. Differences between groups were statistically analyzed using one-way analysis of the variance followed by a Tukey's range test. ${ }^{* *} \mathrm{P}<0.01,{ }^{* * * *} \mathrm{P}<0.001$ vs. control + SAL. VEGF, vascular endothelial growth factor; SAL, saline; DMI, desipramine; FLX, fluoxetine; TIA, tianeptine.

stressed rats experienced decreased spontaneous locomotion and a reduced exploration in the open field, indicated by a 
significant decrease in the total duration of rearing. Stressed rats were observed to spend less time in the center of the field, which may suggest that chronic social stress exposure induced anxiety-related behavior. This tendency to spend less time in the center may be related to changes (either increases or decreases) in hormone levels on the day of the test or the habituation of rats to the stress procedure. Ovarian hormones are important in the regulation of stress responses and behavior $(32,33)$. A limitation of the current study is that the phase of the estrous cycle rats were in at the time of behavioral testing was not taken into account. However, determining this would have required vaginal sampling, which would have affected stress and interfered with the behavioral tests.

The limbic structures, particularly the hippocampus, are considered to be the regions of the brain that are sensitive to chronic social stress and respond to stressors with structural remodeling (34). However, until recently, only a number of studies have investigated the effect of chronic stress on hippocampal VEGF expression $(14,15)$. Previous studies have found that VEGF mRNA is decreased in the granular cell layer of the dentate gyrus in rats subjected to the chronic unpredictable stress (CUS) (14) and in the CA3 region of the ventral hippocampus in rats subjected to chronic mild stress (CMS) (15). Conversely, Kiuchi et al (35) identified no significant differences in hippocampal and plasma VEGF concentrations in rats exposed to CUS compared with unstressed rats. In addition, Elfving et al (36) reported increased VEGF and VEGFR-2 protein levels in the prefrontal cortex, but not in the hippocampus, following chronic restraint stress. A previous suggestion is that the anatomical localization of stress effects on VEGF mRNA and protein expression depends on the applied stress paradigm (37). Chronic social instability stress, used in the present study, differs from the models used in previous studies in regards to its nature and intensity $(38,39)$. This paradigm, designed for female rats (40), is based on repeated but unpredictable homotypic stress of a social nature. CUS and CMS used in previous studies $(14,15)$ on male rats are based on a constant variation in stressors of physical nature, while the restraint stress model is based on exposure to a repeated and predictable homotypic physical stressor. Considering the neuroprotective role of VEGF $(41,42)$, the increase in VEGF mRNA expression in the hippocampus, amygdala and hypothalamus observed following chronic social stress in the present study may be an adaptive response, which develops to maintain the homeostasis of an organism that is impaired by stress. However, it cannot be excluded that this effect is mediated by a stress-induced increase in circulating levels of glucocorticoids (43), which are responsible for the neurobiological effects of stress. The suggestion concerning glucocorticoids is in line with the results of a previous study which showed that long-term corticosterone treatment increased VEGF protein levels in the frontal cortex (44). In the current study, the stress-induced increase in VEGF mRNA expression was largest in the hippocampus, a structure implicated in the pathogenesis of depressive disorders and important for the actions of antidepressants.

The potential role of VEGF in the action of antidepressant drugs has previously been examined $(17,18)$. VEGF was identified to mediate the antidepressant effect of the cAMP cascade in the adult rat hippocampus (45), and that short-term exposure of mice to an enriched environment (a large cage supplied with plastic tubes, ropes, two running wheels, and toys) produced antidepressant-like effects via a VEGF-mediated increase in spinogenesis (46). Furthermore, intracerebroventricular VEGF infusions had an antidepressant effect in behavioral models in rodents (18). In addition, previous studies have determined that VEGF-Flk-1 signaling is required for a number of the behavioral effects (forced swim test, learned helplessness, novelty suppressed feeding) observed in long-term antidepressant treatment (SSRI and serotonin and noradrenaline reuptake inhibitors) in rodents $(17,18)$. In addition, these studies found that DMI (18) and FLX (17) increased VEGF mRNA expression and protein levels in the hippocampus of rodents. Similarly, the present study identified that long-term antidepressant treatment increased VEGF mRNA in the hippocampus of stressed rats, with DMI having the strongest effect. Greene et al (17) observed increased VEGF mRNA levels in the hippocampi of rats subjected to CUS and FLX treatment, but not in rats subjected to CUS alone. It has been suggested that the rapid effects of antidepressants, and increase in VEGF, are mediated by alterations in neuroplasticity and synaptic transmission, whereas prolonged effects are related to enhanced neurogenesis (47).

Interestingly, the present study identified that long-term DMI administration decreased the number of VEGF mRNA copies in the hypothalamus and amygdala in comparison to the rats subjected to chronic social instability stress and treated with saline. In addition, chronic administration of FLX attenuated the stress-induced increase in VEGF mRNA expression in the amygdala and the hypothalamus as compared with the saline-treated stressed rats. The results of DMI treatment may be due to the fact that this antidepressant works primarily by inhibiting the reuptake of norepinephrine (NE) and secondarily by blocking serotonin [5-hydroxytryptamine (5-HT)] reuptake (48). An enhancement of noradrenergic and/or serotonergic system activity is thought to account for the antidepressant action of drugs applied in the therapy of mood disorders (49). Udo et al (50) suggested that VEGF affected the concentration of monoamines in the forebrain of VEGF-overexpressing transgenic mice and that VEGF function was closely linked to the NE system. Udo et al (50) found that levels of NE and 5-HT were reduced exclusively in the regions of the nervous system where VEGF was expressed and that the turnover of NE was markedly increased. Based upon the above findings, it may be suggested that higher levels of the monoamines, particularly $\mathrm{NE}$, induced by long-term antidepressant treatment, are responsible for the decrease in VEGF expression in the amygdala and hypothalamus.

In the current study, TIA was determined to have no effect on VEGF mRNA expression, in all studied brain structures, in chronically stressed rats compared with saline-treated stressed rats. To the best of our knowledge, this is the first study investigating the influence of TIA on the VEGF expression in the rat brain. TIA, an antidepressant with well-known neuroprotective properties (51), does not inhibit the 5-HT or NE reuptake, but normalizes disrupted glutamatergic neurotransmission (52). Previous studies suggest that TIA may promote neuroplasticity in the hippocampus (53) and amygdala (54) by influencing BDNF and nerve growth factor expression. The lack of effect of TIA on VEGF expression in the rats subjected to stress may 
be related to its non-monaminergic mechanism of action. The results of the present study suggest that the neurogenic action of TIA under chronic social stress conditions is not mediated by VEGF.

VEGF has been implicated in a number of neuropsychiatric disorders, however, it remains unclear whether serum VEGF levels reflect the alterations in brain VEGF mRNA or protein levels (55). In the present study markedly reduced serum VEGF protein levels were found compared with VEGF mRNA levels in the studied brain structures in rats exposed to chronic stress, supporting the hypothesis that serum VEGF cannot be considered a marker of stress-induced cerebral alterations. However, the fact that VEGF protein levels in brain structures were not measured limits this conclusion. The decrease in serum VEGF levels observed in the present study is in line with data obtained by Howell et al (44), who found reduced VEGF levels in mice following long-term corticosterone treatment. The studied antidepressants did not reverse the stress-induced decrease in serum VEGF concentration. Similarly to results in the brain structures, DMI and TIA induced opposite effects, DMI increased serum VEGF levels compared with saline-treated stressed rats, whereas TIA reduced VEGF levels compared with saline-treated unstressed control rats. The results of clinical studies concerning plasma VEGF levels in depressed patients are inconsistent (56-61). Lee and Kim (58) reported enhanced plasma VEGF levels in patients with major depressive disorder prior to antidepressant treatment, suggesting that VEGF plasma levels could be used as a biomarker of depression. Similar results were identified in a recent study by Tseng et al (59) based on a meta-analysis involving 16 articles. In contrast, Dome et al (60) and Ventriglia et al (61) found no difference in serum VEGF levels prior to and following antidepressant treatment.

In conclusion, the present study indicates that there is a strong association between the effects of chronic stress, trophic factors and response to antidepressants. In female rats, DMI was identified as the most efficient drug in regards to blocking stress-induced changes in the VEGF mRNA expression in the studied brain structures and serum VEGF protein levels. In addition, the results of the current study suggest that, under chronic stress conditions, VEGF serves a role in the mechanism of action of DMI, which is known to modulate norepinephrine system activity.

\section{Acknowledgements}

The present study was supported by the Medical University of Silesia (grant no. KNW-1-028/D/1/0) and The Jerzy Kukuczka Academy of Physical Education (grant no. F/UP/14/2015), Katowice, Poland.

\section{References}

1. Clark-Raymond A and Halaris A: VEGF and depression: A comprehensive assessment of clinical data. J Psychiatr Res 47: 1080-1087, 2013.

2. Newton SS, Fournier NM and Duman RS: Vascular growth factors in neuropsychiatry. Cell Mol Life Sci 70: 1739-1752, 2013.

3. Brown GW: Social roles, context and evolution in the origins of depression. J Health Soc Behav 43: 255-276, 2002.

4. Warner-Schmidt JL and Duman RS: VEGF as a potential target for therapeutic intervention in depression. Curr Opin Pharmacol 8: 14-19, 2008.
5. Jin K, Zhu Y, Sun Y, Mao XO, Xie L and Greenberg DA: Vascular endothelial growth factor (VEGF) stimulates neurogenesis in vitro and in vivo. Proc Natl Acad Sci USA 99: 11946-11950, 2002.

6. McCloskey DP, Croll SD and Scharfman HE: Depression of synaptic transmission by vascular endothelial growth factor in adult rat hippocampus and evidence for increased efficacy after chronic seizures. J Neurosci 25: 8889-8897, 2005.

7. Khaibullina AA, Rosenstein JM and Krum JM: Vascular endothelial growth factor promotes neurite maturation in primary CNS neuronal cultures. Brain Res Dev 148: 59-68, 2004.

8. Cao R, Eriksson A, Kubo H, Alitalo K, Cao Y and Thyberg J: Comparative evaluation of FGF-2-, VEGF-A-, and VEGF-C-induced angiogenesis, lymphangiogenesis, vascular fenestrations and permeability. Circ Res 94: 664-670, 2004.

9. Fournier NM and Duman RS: Role of vascular endothelial growth factor in adult hippocampal neurogenesis: Implications for the pathophysiology and treatment of depression. Behav Brain Res 227: 440-449, 2012.

10. Nowacka MM and Obuchowicz E: Vascular endothelial growth factor (VEGF) and its role in the central nervous system: A new element in the neurotrophic hypothesis of antidepressant drug action. Neuropeptides 46: 1-10, 2012.

11. Sun R, Li N and Li T: VEGF regulates antidepressant effects of lamotrigine. Eur Neuropsychopharmacology 22: 424-30, 2012.

12. Duman RS and Monteggia LM: A neurotrophic model for stress-related mood disorders. Biol Psychiatry 59: 1116-1127, 2006.

13. Kahl KG, Bens S, Ziegler K, Rudolf S, Kordon A, Dibbelt L and Schweiger U: Angiogenic factors in patients with current major depressive disorder comorbid with borderline personality disorder. Psychoneuroendocrinology 34: 353-357, 2009.

14. Schmidt HD and Duman RS: The role of neurotrophic factors in adult hippocampal neurogenesis, antidepressant treatments and animal models of depressive-like behavior. Behav Pharmacol 18: 391-418, 2007.

15. Heine VM, Zareno J, Maslam S, Joels M and Lucassen PJ: Chronic stress in the adult dentate gyrus reduces cell proliferation near the vasculature and VEGF and Flk-1 protein expression. Eur J Neurosci 21: 1304-1314, 2005.

16. Bergström A, Jayatissa MN, Mørk A and Wiborg O: Stress sensitivity and resilience in the chronic mild stress rat model of depression; an in situ hybridization study. Brain Res 1196: 41-52, 2008.

17. Greene J, Banasr M, Lee B, Warner-Schmidt J and Duman RS: Vascular endothelial growth factor signaling is required for the behavioral actions of antidepressant treatment: Pharmacological and cellular characterization. Neuropsychopharmacology 34: 2459-2468, 2009.

18. Warner-Schmidt JL and Duman RS: VEGF is an essential mediator of the neurogenic and behavioral actions of antidepressants. Proc Natl Acad Sci USA 104: 4647-4652, 2007.

19. Kessler RC: Epidemiology of women and depression. J Affect Disord 74: 5-13, 2003.

20. Kudielka BM and Kirschbaum C: Sex differences in HPA axis responses to stress: A review. Biol Psychol 69: 113-132, 2005.

21. Palanza P: Animal model of anxiety and depression: How are females different? Neurosci Biobehav Rev 25: 219-233, 2001.

22. Directive 2010/63/EU of the European Parliament and of the Council of 22 September 2010 on the protection of animals used for scientific purposes Text with EEA relevance. Official Journal of the European Union, L 276, pp33-pp79, 2010.

23. Nowacka MM, Paul-Samojedny M, Bielecka AM and Obuchowicz E: Chronic social instability stress enhances vulnerability of BDNF response to LPS in the limbic structures of female rats: A protective role of antidepressants. Neurosci Res 88: 73-83, 2014.

24. Paxinos G and Watson C: The Rat Brain in Stereotaxic Coordinates, 6th edition. Academic Press, Sydney, 2007.

25. Nowacka MM, Paul-Samojedny M, Bielecka AM, Plewka D, Czekaj P and Obuchowicz E: LPS reduces BDNF and VEGF expression in the structures of the HPA axis of chronic social stressed female rats. Neuropeptides 54: 17-27, 2015.

26. Chomczynski P: A reagent for the single-step simultaneous isolation of RNA, DNA and proteins from cell and tissue samples. Biotechniques 15: 532-534, 536-537, 1993.

27. Livak KJ and Schmittgen TD: Analysis of relative gene expression data using real-time quantitative PCR and the 2(-Delta Delta C(T)) method. Methods 25: 402-408, 2001. 
28. McEwen BS: Physiology and neurobiology of stress and adaptation: Central role of the brain. Physiol Rev 87: 873-904, 2007.

29. Forbes NF, Stewart CA, Matthews K and Reid IC: Chronic mild stress and sucrose consumption: Validity as a model of depression. Physiol Behav 60: 1481-1484, 1996.

30. Matthews K, Forbes N and Reid IC: Sucrose consumption as an hedonic measure following chronic unpredictable mild stress. Physiol Behav 57: 241-248, 1995.

31. Forbes NF, Stwart CA, Matthews K and Reid IC: Chronic mild stress and sucrose consumption: Validity as a model of depression. Physiol Behav 60: 1481-1484, 1996.

32. Ter Horst GJ, Wichmann R, Gerrits M, Westenbroek C and Lin Y: Sex differences in stress responses: Focus on ovarian hormones. Physiol Behav 97: 239-249, 2009.

33. Sisk CL and Zehr JL: Pubertal hormones organize the adolescent brain and behavior. Front Neuroendocrinol 26 : 163-174, 2005

34. Fuchs E and Flügge G: Chronic social stress: Effects on limbic brain structures. Physiol Behav 79: 417-427, 2003.

35. Kiuchi T, Lee $\mathrm{H}$ and Mikami T: Regular exercise cures depression-like behavior via VEGF-Flk-1 signaling in chronically stressed mice. Neuroscience 207: 208-217, 2012.

36. Elfving B, Jakobsen JL, Madsen JC, Wegener G and Müller HK: Chronic restraint stress increases the protein expression of VEGF and its receptor VEGFR-2 in the prefrontal cortex. Synapse 69: 190-194, 2015.

37. Dioro D, Viau V and Meaney MJ: The role of the medial prefrontal cortex (cingulate gyrus) in the regulation of hypothalamic-pituitary-adrenal responses to stress. J Neurosci 13 3839-3847, 1993.

38. Blanchard DC, Spencer RL, Weiss SM, Blanchard RJ, McEwen BS and Sakai RR: Visible burrow system as a model of chronic social stress: Behavioral and neuroendocrine correlates. Psychoneuroendocrinology 20: 117-1134, 1995.

39. Zelena D, Haller J, Halász J and Makara GB: Social stress of variable intensity: Physiological and behavioral consequences. Brain Res Bull 48: 297-302, 1999.

40. Herzog CJ, Czéh B, Corbach S, Wuttke W, Schulte-Herbrüggen $\mathrm{O}$, Hellweg R, Flügge $\mathrm{G}$ and Fuchs E: Chronic social instability stress in female rats: A potential animal model for female depression. Neuroscience 159: 982-992, 2009.

41. Greenberg DA and Jin K: From angiogenesis and neuropathology. Nature 438: 954-959, 2005.

42. Sanchez A, Wadhwani S and Grammas P: Multiple neurotrophic effects of VEGF on cultured neurons. Neuropeptides 44: $323-331,2010$

43. Conrad C: What is the functional significance of chronic stress-induced CA3 dendritic retraction within the hippocampus? Behav Cogn Neurosci Rev 5: 41-60, 2006.

44. Howell KR, Kutiyanawalla A and Pillai A: Long-term continuous corticosterone treatment decreases VEGF receptor-2 expression in frontal cortex. PloS One 6: e20198, 2011.

45. Lee C and Agoston DV: Inhibition of VEGF receptor 2 increased cell death of dentate hilar neurons after traumatic brain injury. Exp Neurol 220: 400-403, 2009.
46. Huang $\mathrm{YF}$, Yang $\mathrm{CH}$, Huang $\mathrm{CC}$ and Hsu KS: Vascular endothelial growth factor-dependent spinogenesis underlies antidepressant-like effects of enriched environment. J Biol Chem 287: 40938-40955, 2012.

47. PittengerC and Duman RS: Stress, depression, and neuroplasticity: A convergence of mechanism. Neuropsychopharmacology 33 88-109, 2008

48. Gillman PK: Tricyclic antidepressant pharmacology and therapeutic drug interactions updated. Br J Pharmacol 151: 737-748, 2007.

49. Slattery DA, Hudson AL and Nutt DJ: Invited review: The evolution of antidepressant mechanisms. Fundam Clin Pharmacol 18: 1-21, 2004

50. Udo H, Hamasu K, Furuse M and Sugiyama H: VEGF-induced antidepressant effects involve modulation of norepinephrine and serotonin system. Behav Brain Res 275: 107-113, 2014.

51. Uzbay TI: Tianeptine: Potential influences on neuroplasticity and novel pharmacological effects. Prog Neuropsychopharmacol Biol Psychiatry 32: 915-924, 2008.

52. Kasper S and McEwen BS: Neurobiological and clinical effects of the antidepressant tianeptine. CNS Drugs 22: 15-26, 2008.

53. Alfonso J, Frick LR, Silberman DM, Paulumbo ML, Genaro AM and Frasch AC: Regulation of hippocampal gene expression is conserved in two species subjected to different stressors and antidepressant treatments. Biol Psychiatry 59: 244-251, 2006.

54. Reagan LP, Hendry RM, Reznikov LR, Piroli GG, Wood GE, McEwen BS and Grillo CA: Tianeptine increases brain-derived neurotrophic factor expression in the rat amygdala. Eur J Pharmacol 565: 68-75, 2007.

55. Elfving B, Plouqmann PH and Wegener G: Differential brain, but not serum VEGF levels in a genetic rat model of depression. Neurosci Lett 474: 13-16, 2010.

56. Dome P, Teleki Z, Rihmer Z, Peter L, Dobos J, Kenessey I, Tovari J, Timar J, Paku S, Kovacs G and Dome B: Circulating endothelial progenitor cells and depression: A possible novel link between heart and soul. Mol Psychiatry 14: 523-531, 2009.

57. Isung J, Mobarrez F, Nordstrom A, Asberg M and Jokinen J: Low plasma vascular endothelial growth factor (VEGF) associated with completed suicide. World J Biol Psychiatry 13: 468-473, 2011.

58. Lee BH and Kim YK: Increased plasma VEGF levels in major depressive or manic episodes in patients with mood disorders. J Affect Disord 136: 181-184, 2012.

59. Tseng PT, Cheng YS, Chen YW, Wu CK and Lin PY: Increased levels of vascular endothelial growth factor in patients with major depressive disorder: A meta-analysis. Eur Neuropsychopharmacol 25: 1622-1630, 2015.

60. Dome P, Halmai Z, Dobos J, Lazary J, Gonda X, Kenessey I, Sallai T, Makkos Z and Faludi G: Investigation of circulating endothelial progenitor cells and angiogenic and inflammatory cytokines during recovery from an episode of major depression. J Affect Disord 136: 1159-1163, 2012.

61. Ventriglia M,Zanardini R, Pedrini L, Placentino A, Nielsen MG, Gennarelli $\mathrm{M}$ and Bocchio-Chiavetto L: VEGF serum levels in depressed patients during SSRI antidepressant treatment. Prog Neuropsychopharmacol Biol Psychiatry 33: 146-149, 2009. 